\title{
La crisis del lenguaje en La cantatrice chauve
}

\author{
Armando Trinidad AgUILAR DE LEÓN \\ Universidad Nacional Autónoma de México
}

\begin{abstract}
La cantatrice chauve es una obra representativa de una corriente crítica llamada crisis del lenguaje. Para dar a la problemática de la significación y la comunicación un tratamiento espectacular, Ionesco pone en escena a personajes compulsivos, inmersos en un espacio saturado y en un tiempo demencial. Buscando dar a la crisis del lenguaje la máxima proyección escénica, el dramaturgo introduce en el diálogo mismo un elemento —el fuego- que será definitivo para el efecto final de la obra: la desarticulación de la palabra.
\end{abstract}

PALABRAS ClAVE: crisis del lenguaje, significación, comunicación, lenguaje escénico, absurdo.

La cantatrice chauve represents the critical movement currently known as Crisis of language. In order to treat meaning and communication in a spectacular manner, Ionesco places on stage several compulsive characters, immersed in a saturated space and in an insane time. In his attempt to give the Crisis of language the strongest scenic projection, the author introduces an element - fire-into the dialogue itself, which will determine the final effect of the play: the disassembling of the speech.

KEY WORDS: crisis of language, meaning, communication, scenic language, absurd.

Oh Dear, what non-sense I'm talking!

LEWIS CARROLL

Testimonio de una época turbulenta en la que el ser humano revaloriza su entorno y su ser mismo, la obra La cantatrice chauve, de Eugène Ionesco, participa en la discusión que el pensamiento del siglo Xx tiene sobre el lenguaje. La ciencia y el arte enfocan, entonces, la comunicación. La lingüística centra el acto del habla y se da a la tarea de describir minuciosamente el circuito comunicativo; al hacerlo, deja expuesto el proceso de significación y revela las funciones del lenguaje. Diversas disciplinas aprovechan estos hallazgos. Por una parte, el psicoanálisis desentraña del acto verbal las causas de la conducta del individuo, la sociolingüística se interesa en la interacción 
entre los miembros de un grupo, la semiótica observa toda producción humana como un signo (fusión de significado y significante) con funciones específicas en el seno de la vida social... Por otra parte, la pintura propone técnicas revolucionarias que fundan un nuevo lenguaje plástico y la literatura emprende una búsqueda en la que la palabra y sus atributos saldrán en mejor o peor estado. El teatro se encuentra, también, en una revisión del lenguaje escénico.

En este panorama se sitúa la obra de Eugène Ionesco. En una época en la que se analiza el proceso de significación y se diserta sobre el acto comunicativo, Ionesco ancla ambos asuntos en la inmediatez de la realidad cotidiana y aporta argumentos contundentes a la crítica del lenguaje. Su experiencia en el aprendizaje de una lengua extranjera —el inglés - y su observación de la vida diaria le hacen tomar conciencia del sinsentido y de la actitud del ser humano en la comunicación. Luego, traslada sus reflexiones al escenario y pone bajo los reflectores a personajes sorprendentes, inmersos en situaciones 'insólitas' de interlocución: "l'insolite ne peut surgir, à mon avis, que du plus terne, du plus quelconque quotidien, de la prose de tous les jours", dice Ionesco (1991: 229). Agreguemos a ello la concepción que él tiene del teatro: "Le théâtre est dans l'exagération extrême des sentiments, exagération qui disloque la plate réalité quotidienne. Dislocation aussi, désarticulation du langage" (ibid.: 60).

Los aspectos mencionados son, precisamente, la base de la construcción dramática de La cantatrice chauve. En ella, Ionesco presenta el acto comunicativo como un fenómeno fallido. Sus personajes, atrapados en un espacio opresivo y en un tiempo dislocado, hablan compulsivamente; el lenguaje es sólo un punto de interacción social, una serie de réplicas desgastadas, de sonidos sin significado; el diálogo avanza de forma accidentada hacia el paroxismo verbal; al final, la palabra yace, 'literalmente', desarticulada en escena (infra: "IV. El fuego y su papel en la desarticulación verbal"). Para lograr tal efecto, Ionesco hace que los elementos dramáticos se respondan con un mismo fin: escenificar la crisis del lenguaje.

Habrá que notar que el asunto central de La cantatrice chauve viene anunciándose desde las primeras propuestas del teatro de vanguardia. En efecto, desde Jarry, puede trazarse una clara trayectoria del tema que encuentra en el teatro de Ionesco su expresión más hilarante.

En las páginas siguientes, desarrollaremos lo anterior. El primer apartado explora la producción de vanguardia y del absurdo que plantean sus respectivas reflexiones sobre la palabra en el lenguaje escénico y en el ejercicio cotidiano de la comunicación. En un segundo momento, se aborda el proceso de creación de la antipieza y las reacciones del público en ocasión del estreno de la obra. La destrucción del tiempo y del espacio es el tema del tercer apartado, mientras que el cuarto y último está dedicado al papel que tiene el fuego a lo largo de La cantatrice chauve. 


\section{La palabra en el lenguaje escénico}

En el siglo XX el cuestionamiento sobre el lenguaje ${ }^{1}$ encuentra las condiciones adecuadas para manifestarse con mayor intensidad y los adelantos tecnológicos necesarios para su amplia difusión. La crítica del lenguaje irriga entonces diferentes campos del conocimiento en dos grandes tendencias: la primera, descriptiva, que se encarga de exponer los procesos propios de la significación y de la comunicación; la segunda, de tendencia pragmática, que observa un mal funcionamiento del mecanismo básico del lenguaje — la significación — que afecta el objetivo primordial de éste: la comunicación. Interesado en este último fenómeno, George Steiner precisa que lo que está en duda son "los conceptos mismos de significado [y] la posibilidad de relaciones significantes entre la palabra y el mundo" (1992: 110). Se habla, luego, de una fisura en el signo, de una ruptura entre el significante y el significado, de una pérdida de sentido, de una no correspondencia entre las palabras y su referente, de la incapacidad del ser humano frente al tipo de lenguaje inherente a su especie.

Al respecto, el teatro toma una postura radical y contribuye a la difusión de dicha crisis. En Francia, los dramaturgos de vanguardia, Alfred Jarry, Guillaume Apollinaire y Antonin Artaud, proponen un tratamiento innovador del lenguaje escénico que viene a dar al lenguaje verbal una proyección espectacular, destacando, precisamente, su uso cotidiano.

Para Jarry, por ejemplo, el espectáculo consiste en una fusión de los elementos dramáticos: la escenografía, la iluminación, el vestuario, la interpretación, los efectos sonoros...; en esta larga enumeración se encuentra, por supuesto, la palabra y la voz: "Il va sans dire qu'il faut que l'acteur ait une voix spéciale" (2000: 313). En el estreno de Ubu roi (1896), los protagonistas portan una máscara que presiona sus fosas nasales; esto produce un efecto especial en la pronunciación. De entrada, la primera palabra de la obra: "Merdre", pronunciada en un tono nasal, con una ' $r$ ' de más, provoca en los espectadores una carcajada que se convierte en hilaridad conforme se incrementan las intervenciones de los personajes, el titular dice: "J'ai des oneilles pour parler et vous une bouche pour m'entendre" (ibid.: 83). Frente a esta forma de articular y a este tipo de confusiones, el público reacciona: grita, aúlla, silba y lanza proyectiles. Jarry se propuso "que la pièce ne pût aller jusqu'au bout et que le théâtre éclatât" (ibid.: 433-437).

Casi veinte años después, Guillaume Apollinaire, también en pos del espectáculo, fusiona gestos, sonidos, ruidos, gritos, colores, música y explota la capacidad escénica de la palabra. En Les Mamelles de Tirésias² (1917), los personajes gritan al público

\footnotetext{
${ }^{1}$ El cuestionamiento sobre el lenguaje se remonta hasta la filosofía de Platón; el Crátilo es, de hecho, el primer punto de referencia de esa postura que en el siglo XX es llamada 'crisis del lenguaje'.

${ }^{2}$ Calificada como "le grand événement de l'avant-garde en 1917" (Décaudin en Apollinaire, 2000: 10), Les Mammelles de Tirésias tiene la precisión genérica de "Drame surréaliste", inventada por Apollinaire para la ocasión; así, Apollinaire se sitúa en la vanguardia de una nueva expresión artística que se extenderá
} 


\section{$110 \square$ LA CRISIS DEL LENGUAJE EN LA CANTATRICE CHAUVE}

algunas de sus réplicas sirviéndose de un altavoz. El marido de Thérèse, la protagonista, habla con un acento marcado. A veces, ambos producen onomatopeyas sin sentido: "Elle sort en caquetant tandis que le mari imite le bruit de la locomotive en marche" (ibid.: 127).

Más tarde, en los años treintas, Antonin Artaud insiste en la idea de restituir a la puesta en escena su naturaleza espectacular. Al ser el teatro un espectáculo el aspecto visual de la puesta en escena viene a ocupar un lugar determinante en la construcción dramática. Artaud propone quitar peso a los diálogos para explotar otros medios de expresión: la pantomima, la música, la danza y la iluminación, entre otros: "Le dialogue —chose écrite et parlée — n'appartient pas spécifiquement à la scène” (1999: 55). El dramaturgo desconfía abiertamente de la palabra: "je pose en principe que les mots ne veulent tout dire [...], ils arrêtent et paralysent la pensée" (ibid.: 171-172).

El teatro de la década de los cuarentas presenta una notable diferencia con relación a las propuestas de proyección escénica del teatro precedente. El lenguaje verbal se convierte en un punto de reflexión para los personajes mismos, que se encuentran en un momento difícil de su 'existencia'. En Huis clos (1944), Garcin pide a sus compañeras de celda: "du silence. Pas un mot. [...] Et nous... nous serons sauvés" (Sartre, 1994: 42). Jan, personaje de Le Malentendu (1944), suplica: “O mon Dieu! Donnezmoi de trouver mes mots ou faites que j'abandonne cette vaine entreprise" (Camus, 2001: 209); en Médée (1946), el Jasón de Jean Anouilh dice: "Les mots ne sont rien, mais il faut qu'ils soient dits tout de même" (1998: 62).

Paralelo a estas propuestas dramáticas se desarrolla un pensamiento filosófico que destaca lo absurdo de la existencia humana. En 1942, en "Les murs absurdes", ${ }^{3}$ Albert Camus reflexiona sobre un mundo 'espeso' y 'extraño' — “cette épaisseur et cette étrangeté du monde, c'est l'absurde” (2000: 31) — donde los seres humanos actúan y hablan como autómatas: "l'aspect mécanique de leurs gestes, leur pantomime privée de sens rend stupide tout ce qui les entoure" (idem). Según Camus, el hombre está atrapado en los muros de un "univers indicible où règnent la contradiction, l'antonymie, l'angoisse ou l'impuissance" (ibid.: 41). Camus establece una relación directa entre el acto del habla (gestos mecánicos, contradicción, antonimia) y el estado emocional del ser humano (la angustia y la impotencia).

En las líneas trazadas por el teatro de vanguardia y por la filosofía del absurdo, el teatro de los años cincuentas - conocido como teatro del absurdo o teatro de lo irrisorio (Jacquart, 1998) — presenta el problema de la crisis del lenguaje en situaciones cotidianas de interlocución. Los dramaturgos proyectan en escena el desempeño de las personas en la comunicación. Marie-Claude Hubert observa: "De nombreux dramatur-

en pocos años a las diversas artes del hemisferio occidental. André Breton, en su primer manifiesto (1924), lo saluda como el precursor del movimiento que decide nombrar surréalisme: "En hommage à Guillaume Apollinare qui venait de mourir" (1999: 35).

${ }^{3}$ En 1942 Camus publica Le mythe de Sisyphe. Un essai sur l'absurde; "Les murs absurdes" forma parte de este ensayo. 
ges poursuivent alors une réflexion permanente sur le langage [...]. Leurs héros sont murés dans le silence ou dans de longs monologues [...], englués dans des conversations où les mots n'ont guère plus de valeur qu'une balle qu'ils se renvoient pour meubler le vide de leur existence, pour maintenir un dérisoire contact" (1987: 11-12).

Tal observación puede ser aplicada al uso de la palabra en el teatro de Ionesco. Como sus predecesores, eI dramaturgo rumano-francés reflexiona sobre el lugar de la palabra en el lenguaje escénico: "Le théâtre a une façon propre d'utiliser la parole, c'est le dialogue" (1991: 62). Si el diálogo es uno de los tantos medios de expresión del teatro, la palabra es un procedimiento más en la puesta en escena: "la parole ne constitue qu'un des éléments de choc du théâtre" (idem). Ionesco reflexiona también sobre el valor del arte dramático, el cual radica en la amplificación de sus diversos elementos: "Si donc la valeur du théâtre était dans le grossissement des effets, il fallait les grossir encore, les souligner, les accentuer au maximum" (ibid.: 59).

Al ser la palabra uno de los medios de expresión del teatro, Ionesco la trabaja a su manera: en La cantatrice chauve crea una atmósfera extraña, con un espacio opresivo y un tiempo demencial, donde interactúan personajes ordinarios, enredados en las peripecias de la conversación. El diálogo es una serie de réplicas incoherentes en las que el sentido y la referencia se pierden en una proliferación de términos inútiles, de frases parásitas. Ionesco aprovecha al máximo la proyección escénica de la palabra y la hace, incluso, explotar en sus elementos mínimos: "Le verbe lui-même doit être tendu jusqu'à ses limites, le langage doit presque exploser, ou se détruire, dans son impossibilité de contenir les significations" (63).

Esta intención de Ionesco de dramatizar la crisis de lenguaje es consecuencia de su experiencia inmediata con los problemas de significación y de comunicación, así como de un trabajo de producción minucioso, cuyas grandes líneas presentamos a continuación.

\section{La cantatrice chauve: producción y recepción}

La génesis de La cantatrice chauve se encuentra en la curiosidad de Ionesco por el idioma inglés. Entregado a la tarea de aprenderlo, Ionesco descubre en su manual, L'Anglais sans peine del método Assimil, la materia prima de su obra: las lecciones de inglés lo enfrentan a frases manipuladas con fines didácticos pero que expresaban 'verdades universales y antagónicas', como "il y a sept jours dans la semaine" o "le plancher est en bas, le plafond en haut" (ibid.: 243-245); frente a estos hechos indiscutibles, Ionesco dice: "J'eus une illumination. Il ne s'agissait plus pour moi de parfaire la connaissance de la langue anglaise. [...] Mon ambition était devenue plus grande: communiquer à mes contemporains les vérités essentielles dont m'avait fait prendre conscience le manuel de conversation franco-anglaise" (ibid.).

L'Anglais sans peine le proporciona, además, cinco de sus personajes: los matrimonios Smith y Martin, y Mary, la bonne: "les Smith et les Martin du manuel sont les 
Smith et les Martin de ma pièce, ils sont les mêmes, prononcent les mêmes sentences, font les mêmes actions et les mêmes "inactions"” (ibid.: 246). Si bien Ionesco no da mayor precisión sobre el origen de la bonne, Martine Cécillon, en su "Lecture accompagnée de La cantatrice chauve", incluye una ilustración del libro de inglés en donde se ve una sirvienta que afirma "I had a pleasant evening!" (1998: 45); declaración que es, precisamente, la primera intervención de Mary ( $c f$. Ionesco, 1954: 51), el quinto personaje de la obra. A estos personajes, Ionesco agrega un capitán de bomberos.

La conversación entre ellos es una dramatización de los diálogos didácticos: "Toute une partie de la pièce est faite de la mise à bout des phrases extraites de mon manuel d'anglais" (246); sólo que los diálogos, las situaciones del libro y los mismos personajes tienen relación con la realidad cotidiana, la cual Ionesco observa con disgusto: "je regarde et je vois des images, des êtres qui se meuvent, dans un temps sans temps, dans un espace sans espace, imitant de sons qui sont une sorte de langage que je ne comprends plus" (1991: 193). Estos seres extraños son un tipo social, le petit bourgeois, que Ionesco compara con cierta ave, célebre por hablar por imitación y no saber lo que dice: "Le perroquet $[\ldots]$. Ce qu'il dit ne le concerne pas, [...] ne le comprend pas" (ibid.: 29). Esta 'pequeña burguesía' — compuesta por seres humanos promedio, de cualquier posición económica, en cualquier sociedad - se encarna en los Smith y los Martin, personajes de L'Anglais sans peine. Ionesco plantea, entonces, desde diferentes aspectos, un paralelo entre su libro de inglés y la inmediata realidad cotidiana. Esto revela los lazos hipertextuales y contextuales que alimentan la primera obra de Ionesco, cuyo título no estaba aún definido.

Al respecto, Ionesco dudaba entre algunas variantes de L'Anglais sans peine: L'Heure anglaise, Big-Ben Folies, Une Heure d'anglais, etcétera, pero "les connotations trop britanniques risquaient de dénaturer le sens de la pièce" (Cécillon en Ionesco, 1998: 43). Ionesco dice que la casualidad intervino en la elección del título: en uno de los ensayos, el actor que representaba al capitán de bomberos, en un lapsus linguae, confunde una institutrice blonde - escrito en el guión - con una cantatrice chauve: "Voilà le titre de la pièce!", gritó Ionesco (1991: 253). Puesto que en el proceso de producción de la obra Ionesco tiene en mente la crisis del lenguaje, resulta por demás 'curioso' que un accidente lingüístico haya venido a determinar su título.

Al título, 'dictado por el azar', Ionesco agrega la precisión genérica anti-pièce; ésta previene que la construcción dramática no corresponde a la estructura tradicional de las obras de teatro: "La mention 'anti-pièce', destinée à nous donner des indications génériques, avoue la volonté de Ionesco de rompre avec le théâtre classique en s'y opposant" (Cécillon en Ionesco, 1998: 44). En efecto, Ionesco se opone al teatro clásico no sólo en la configuración de los personajes, también en la recreación de una atmósfera absurda, que resulta de un espacio cerrado y saturado de objetos: "Intérieur bourgeois anglais, avec des fauteuils anglais. [...] M. Smith, Anglais, fume sa pipe anglaise et lit un journal anglais, près d'un feu anglais. [...] Mme Smith, Anglaise, raccommode des chaussettes anglaises. [...] silence anglais. [...] pendule anglaise" (1954: 41), y de un tiempo que se encuentra, simplemente, dislocado: "La pendule 
sonne sept fois. Silence. La pendule sonne trois fois. Silence. La pendule ne sonne aucune fois", otras veces: "La pendule sonne tant qu'elle veut" (ibid.: 46-62).

Al parecer, nada sucede en este escenario lleno y 'anacrónico', nada más que una interacción verbal tan absurda como el espacio opresivo y el tiempo demencial, que, a su vez, resultan ser una proyección de la psique de los personajes ( $c f$. Cécillon en Ionesco, 1998: 43). En un juego de espejos, el diálogo refleja el mundo externo e interno de los interlocutores: "il est évident que nos personnages sont fous, malheureux, perdus, stupides, conventionnels [...], leur parler est absurde, [...] leur langage est désagrégé, comme leur pensée" (Ionesco, 1991: 328).

En boca de estos seres perturbados, el tratamiento de la palabra cobra relevancia. Las indicaciones escénicas detallan el tono de la voz, el titubeo de los interlocutores, sus ademanes mecánicos, el ritmo de las frases... Así, las once escenas irregulares que componen la obra encadenan una secuencia de episodios disparatados. En cada uno de ellos, la iluminación, los efectos sonoros, el desempeño de los actores... se encuentran al servicio de la acción; ésta, de acuerdo con Martine Cécillon, radica en el crescendo verbal (1998: 88).

El día del estreno de La cantatrice chauve, 11 de mayo de 1950, la 'anti-pièce' dirigida por Nicolas Bataille suscita desconcierto. Al final del espectáculo, el grueso del público comenta la puesta en escena; dos personas, habituadas a identificar por el título al protagonista, se preguntan intrigadas:

-Aucune cantatrice n'est apparue, me semble-t-il, ma bonne amie?

-Au moins je ne l'ai pas remarquée. Et chauve! Avez-vous vu que quelqu'un fût chauve?... Et ce pompier? Que vient faire là un pompier?

(Lemarchand en Ionesco, 1954: 10)

Ionesco promete un personaje con características específicas pero "point de cantatrice dans la distribution, pas de calvitie annoncée" (Cécillon en Ionesco, 1998: 43); contrariamente a sus espectativas, el público observó en escena a dos matrimonios autómatas, a una sirvienta perspicaz y a un capitán de bomberos — sólo en aparienciafuera de lugar.

Los espectadores eruditos tampoco pudieron quedarse callados. Una periodista, Renée Sorel, escribió sobre la representación: “Rien n’arrive, personne n'a rien à dire, c'est tout à fait comme dans la vie. Cette anti-pièce de M. E. Ionesco serait en somme entachée du plus vilain naturalisme si le dialogue n'avait une fantaisie parfaitement bouffonne. Il n'y a pas de situation, pas d'action, les entrées et les sorties ne sont nullement motivées, chacun parle sans espoir d'être écouté, ni compris" (1991: 17).

En Notes et contre-notes, el mismo Ionesco cita a algunos de sus críticos. Entre ellos sobresale el inglés Kenneth Tynan, quien escribe en las páginas del l'Observer (1958) el siguiente comentario: “[M. Ionesco] déclarait que les mots n'avaient pas de sens, que la communication entre les hommes était impossible. [II] a créé un monde de robots solitaires, conversant entre eux en dialogues [...] parfois désopilants, 


\section{$114 \square$ LA CRISIS DEL LENGUAJE EN LA CANTATRICE CHAUVE}

parfois évocateurs, souvent ni l'un ni l'autre, et qui, alors, distillent un profond ennui'" (ibid.: 136-137). ${ }^{4}$

De esta forma, tanto el público aficionado como el público especializado participan en la difusión del tema central de la obra: la crisis del lenguaje o el absurdo verbal.

\section{La destrucción del espacio y el tiempo}

En los apartados precedentes quedó asentado que en la concepción dramática de Eugène Ionesco la palabra tiene un tratamiento especial. Ionesco aprovecha sus posibilidades escénicas con el fin de exponer frente al público los alcances de la crisis del lenguaje. Así, Ionesco hace que sus personajes se vean atacados por la más implacable compulsión: se enredan en frases, en sílabas, en fonemas, incluso. Todo esto es consecuencia de la carga emocional que provoca la conversación y del tiempo y el espacio demenciales. En estas condiciones de comunicación la interacción social trae consigo diferentes reacciones anímicas. Los personajes se alteran, se relajan, se vuelven a alterar, hasta perder el poco control de sí mismos. ${ }^{5}$

Tanto la inestabilidad emocional como el tratamiento del tiempo y del espacio afectan el ritmo del diálogo que, a su vez, refleja el mundo interno de los personajes y la extraña atmósfera en la que se encuentran inmersos. Ionesco dice: "J'ai essayé [...] d'extérioriser l'angoisse [...] de mes personnages dans les objets, de faire parler les décors, de visualiser l'action scénique, de donner des images concrètes [...] de l'aliénation" (ibid.: 156).

Para lograr la correspondencia entre la escenografía y el diálogo, Ionesco manipula los recursos verbales: disloca las asociaciones semánticas y fractura la sintaxis. Las estructuras lingüísticas y las relaciones lógicas revelan que el discurso de los Smith y los Martin es una acumulación de sinsentidos, la destrucción de las referencias espaciales y temporales en el diálogo refuerza el tratamiento del espacio y del tiempo escénicos.

Más allá de la destrucción espacio/temporal, cada frase de los personajes revela "la destruction continuelle de tout ce qui se construit" (ibid.: 194). Los seres humanos se diluyen también en una proliferación de referentes que imposibilitan la identificación del individuo, como sucede con Bobby Watson, "impossible d'identifier, car [...] les trois quarts des habitants de la ville, hommes, femmes, enfants, chats, idéologues, portaient le nom de Bobby Watson" (ibid.: 247).

En una obra cuya fuerza radica en la dimensión dramática de la crisis del lenguaje, Ionesco introduce en el discurso mismo el elemento definitivo para el desenlace. A

\footnotetext{
${ }^{4}$ Tynan se refiere no sólo a La cantatrice chauve (1950), también a La Leçon (1951) y a Les Chaises (1952)

${ }^{5}$ De hecho, las variaciones en la expresión verbal y en los gestos representan la parte más dinámica de la puesta en escena.
} 
través del sinsentido y de la destrucción de las referencias, el diálogo avanza hacia la desarticulación de la palabra.

\section{El fuego y su papel en la desarticulación verbal}

Es ahora cuando el fuego reclama su importancia escénica y justifica la inclusión del sexto personaje: el Capitán de bomberos, vinculado, por supuesto, al elemento ígneo. Éste forma parte de la escenografía - Ionesco nos hace saber de su presencia desde la primera indicación escénica- - y aparece en el diálogo con frecuencia, dando siempre un nuevo impulso a la conversación y alterando el ánimo de los personajes.

Más allá del simbolismo inmediato del calor del hogar, de la calidez de las relaciones, el fuego se presenta en La cantatrice chauve con su característica primitiva: su cualidad de combustión. Sin profundizar en el imaginario de Ionesco, hagamos un breve paréntesis: el fuego está presente desde su temprana obra. Ionesco dice haber escrito a los diez años un pequeño libreto: “J'imaginai un goûter d'enfants, troublé par les parents mécontents de constater du désordre. Les enfants, rendus furieux, cassaient la vaisselle, jetaient les parents par la fenêtre et finissaient par mettre feu à la maison" (Ionesco en Philippe Sellier, 1998: 439).

El libreto de infancia presenta elementos que forman parte del universo delirante de La cantatrice chauve: la interacción social, el desorden, la furia, la destrucción, la agresión, el fuego.

En efecto, en esta obra, el fuego tiene un papel decisivo en el desarrollo y en el desenlace de la acción. En la primera escena: "M. Smith, Anglais, [...] fume sa pipe anglaise [...], près d'un feu anglais" (1954: 41), mientras su esposa habla y habla sobre la comida. El tema en cuestión se insinúa a través de su largo monólogo: la acción del fuego está relacionada con los deberes cotidianos de la sirvienta: "Mme Smith: [...] Mary a bien cuit les pommes de terre, cette fois-ci. La dernière fois elle ne les avait pas bien fait cuire. Je ne les aime que lorsqu'elles sont bien cuites" (ibid.: 42).

Conforme la palabra se tensa y el estado de ánimo de los personajes se torna febril, las alusiones al fuego se suceden. Después de la primera discusión (Escena I), M. Smith, busca reconciliarse con su esposa y le pregunta tiernamente: "Oh! Mon petit poulet rôti, pourquoi craches-tu du feu?" (ibid.: 51).

En la Escena VII, las interrupciones constantes ‘encienden’ el ánimo de los Smith y los Martin. A la llegada del Capitán de bomberos (Escena VIII), el diálogo se centra, precisamente, en el tema de la combustión: el bombero pregunta a los anfitriones si, por casualidad, hay algo en esa casa que pueda arder. Ellos niegan, hesitantes. Él insiste: "Rien du tout? Vous n'auriez pas un petit feu de cheminée, quelque chose qui brûle dans le grenier ou dans la cave? Un petit début d'incendie, au moins?" (idem).

Ante la negativa de los Smith, pregunta a los Martin: "Et chez vous, ça ne brûle pas non plus?" (ibid.: 77). Después de lamentar la (supuesta) ausencia de fuego, los matrimonios recomiendan: 


\section{$116 \square$ LA CRISIS DEL LENGUAJE EN LA CANTATRICE CHAUVE}

Mme SмiтH: Est-ce que vous êtes allé voir chez le marchand d'allumettes?

[...]

M. MARTIN: Allez donc voir, de ma part, le vicaire de Wakefield! ${ }^{6}$

El Capitán explica: “Je n'ai pas le droit d'éteindre le feu chez les prêtres. [...] Ils éteignent leurs feux tout seuls ou bien ils le font éteindre par des vestales" (ibid.: 78).

Más tarde, el bombero se resiste a contar una última anécdota; la concurrencia suplica: "Vous avez un cœur de glace. Nous sommes sur des charbons ardents" (ibid.: 84). En tal 'incandescencia', Mary viene a propagar el fuego con un poema, 'incendiario', “qui s'intitule 'Le Feu'”, en honor al Capitán de bomberos:

Les polycandres ${ }^{7}$ brillaient dans les bois

Une pierre prit feu

Le château prit feu

La forêt prit feu

Les hommes prirent feu

Les femmes prirent feu

$[\ldots]$

L'eau prit feu

Le ciel prit feu

La cendre prit feu

La fumée prit feu

Le feu prit feu

Tout prit feu

Prit feu, prit feu (ibid.: 90-91).

Después del poema, el bombero parte al otro extremo de la ciudad en pos del deber. Entonces, los Smith y los Martin llegan al colmo de la exasperación; en el paroxismo, la acción alcanza el clímax: la palabra misma es presa del fuego:

Kakatoes, kakatoes, kakatoes, kakatoes, kakatoes, kakatoes, kakatoes, kakatoes, kakatoes, kakatoes.

Quelle cacade, quelle cacade, quelle cacade, quelle cacade, quelle cacade, quelle cacade, quelle cacade, quelle cacade, quelle cacade (ibid.: 96).

Para el efecto final, Ionesco se vale de recursos fonéticos: las aliteraciones y el ritmo evocan el sonido del fuego que devora: "Les cacaoyers des cacaoyères donnent pas de cacahuètes, donnent du cacao! Les cacaoyers des cacaoyères donnent pas de cacahuètes, donnent du cacao! Les cacaoyers des cacaoyères donnent pas de cacahuètes, donnent du cacao!" (ibid.: 97).

${ }^{6} \mathrm{El}$ 'Vicario de Wakefield' refiere a una novela de Oliver Goldsmith — The Vicar of Wakefield (1766). El protagonista, al volver a su casa, se encuentra que ésta ha sido devorada por el fuego ( $c f$. Jacquart, 1998: 143).

${ }^{7}$ Emanuel Jacquart explica que "policandros" es un neologismo de Ionesco construido sobre las raíces “poli”, muchos, y “candere”, arder, inflamarse' (cf. 1998: 144). 
La palabra, inflamada, termina por explotar en sus elementos fonéticos:

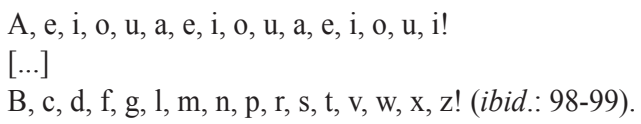

En eso, "La lumière s'est éteinte". En la oscuridad, la palabra crepita sobre le escenario, “C'est pas par là, c'est par ici, c'est pas par là, c'est par ici, c'est pas par là, c'est par ici, c'est pas par là, c'est par ici, c'est pas par là, c'est par ici, c'est pas par là, c'est par ici!" (idem).

El fuego se extingue: "Les paroles cessent brusquement" (ibid.: 100). Entonces, la luz se hace y la obra comienza una vez más, sólo que esta vez los Martin ocupan el lugar de los Smith y dicen exactamente las mismas réplicas de la primera escena. "le rideau se ferme doucement" (idem).

Con la peripecia del intercambio de los Smith por los Martin Ionesco sugiere que los interlocutores de la obra y el mismo lenguaje verbal se encuentran atrapados en un circuito absurdo, como absurda es también la existencia del ser humano promedio, incapaz de servirse de la palabra como una herramienta efectiva en la interacción cotidiana.

La construcción circular de la obra está sostenida por un entramado de procedimientos escénicos que se responden para producir el mismo efecto: amplificar el problema de la crisis del lenguaje. Ionesco lleva este asunto más allá de los límites de la significación y de la comunicación. En La cantatrice chauve, el espectáculo es, en sí, las contorsiones de la palabra que, al correr del diálogo y por la acción del fuego, avanza hacia su desarticulación: "l'explosion finale pulvérise le langage" (Jacquart, 1998: 27).

\section{Obras citadas}

Anouilh, Jean. 1998. Médée. París: La table ronde. (La petite vermillon)

ApollinAIre, Guillaume. 2000. Les Mamelles de Tirésias. París: Gallimard. (Poésie)

ARTAUD, Antonin. 1999. Le théâtre et son double. París: Gallimard. (Folio / Essais) Breton, André. 1999. Manifestes du surréalisme. París: Gallimard.

CAmus, Albert. 2001. Caligula suivi de Le malentendu. Nouvelles versions. París: Gallimard. (Folio)

2000. Le mythe de Sisyphe. París: Gallimard. (Folio / Essais)

HuBERT, Marie-Claude. 1987. Langage et corps fantasmé dans le théâtre des années cinquante. Introd. de Eugène IoNESCO. París: José Corti.

IONESCO, Eugène. 1998. La cantatrice chauve. Lecture accompagnée par Martine Cécillon. París: Gallimard. (La bibliothèque) 
1993. La cantatrice chauve. Edición presentada y anotada por Emmanuel JACQUART. París: Gallimard. (Folio / Théâtre)

1991. Notes et contre-notes. París: Gallimard. (Folio / Essais)

1954. Théâtre I. Prefacio de Jacques Lemarchand. París: Gallimard.

JACQUART, Emmanuel. 1998. Le théâtre de dérision. París: Gallimard. (Tel)

JARRY, Alfred. 2000. Ubu. París: Gallimard. (Folio classique)

SARTre, Jean-Paul. 1994. Huis clos suivi de Les mouches. París: Gallimard. (Folio)

SEllier, Philippe. 1998. "L'effondrement du langage". Dictionnaire du Théâtre.

París: Albin Michel. (Encyclopaedia Universalis)

SteINER, George. 1992. Presencias reales. ¿Hay algo en lo que decimos? Barcelona: Destino. (Ensayos) 